\title{
Autopercepción de la voz en Mujeres Trans de la Región Metropolitana: primer estudio chileno
}

\author{
Carolina Orellana Venegas a , Fabiola Marín Garrido ${ }^{\mathbf{a}}$, Luciana Daniela Muñoz ${ }^{\mathbf{b}, \mathbf{c}, *}$ \\ a Carrera de Fonoaudiología, Facultad de Medicina, Universidad del Desarrollo - Clínica Alemana, Chile \\ bala UAPORRINO, Chile \\ ${ }^{\mathrm{c}}$ CESFAM Dr. Luis Ferrada Urzúa, Chile
}

\section{RESUMEN}

A nivel internacional se reconoce que las mujeres transgéneros, debido a su voz, experimentan diariamente consecuencias psicosociales. En Chile no se cuenta con antecedentes sobre esta temática, por lo que se requiere recabar información que permita constatar los antecedentes obtenidos en la literatura internacional. Por ello, el objetivo del trabajo es describir la autopercepción de la voz de un grupo de mujeres transgénero de la Región Metropolitana, según el instrumento Trans Woman Voice Questionnaire (TWVQ). Para ello, se realizó un estudio observacional, descriptivo correlacional de corte transversal con enfoque cuantitativo, el cual fue aprobado por el Comité de Ética de la Facultad de Medicina - Clínica Alemana Universidad del Desarrollo (GP-70/2018). Participaron 30 mujeres transgénero que respondieron en primer lugar un cuestionario de antecedentes clínicos y luego el TWVQ. Este último se analizó en base al puntaje total y por sus tres ítems (ansiedad y evitación, función vocal e identidad vocal). Los resultados muestran que la autopercepción de la voz se ve afectada en primer lugar por el ítem de función vocal, posteriormente por el ítem de ansiedad y evitación y por último por el de identidad vocal, según la dificultad percibida. Los datos también proporcionan evidencia preliminar de que la autopercepción de la voz podría asociarse con la asistencia al especialista de la voz y con el tiempo de terapia hormonal cursado. Finalmente, el estudio proporciona una forma empírica de analizar detalladamente el TVWQ, en base a la propuesta de tres ítems.

\section{Self-perception of voice in Spanish-speaking Trans Women in Santiago: first Chilean study}

\begin{abstract}
At the international level, it is recognized that transgender women, due to their voice, experience psychosocial consequences on a daily basis. In Chile there is no background on this issue, so it is necessary to collect information that allows verifying the background obtained in the international literature. Therefore, the objective of the work is to describe the self-perception of the voice of a group of transgender women from the Metropolitan Region, according to the Trans Woman Voice Questionnaire (TWVQ) instrument. For this, an observational, descriptive correlational cross-sectional study with a quantitative approach was carried out, which was approved by the Ethics Committee of the Faculty of Medicine - Clínica Alemana Universidad del Desarrollo (GP-70/2018). Thirty transgender women participated, responding first to a medical history questionnaire and then to the TWVQ. The latter was analyzed based on the total score and its three items (anxiety and avoidance, vocal function and vocal identity). The results show that voice self-perception is affected first by the vocal function item, then by the anxiety and avoidance item, and finally by the vocal identity item, according to perceived difficulty. The data also provide preliminary evidence that self-perception of the voice could be associated with attendance at the voice specialist and with the length of time on hormonal therapy. Finally, the study provides an empirical way to analyze the TVWQ in detail, based on the proposal of three items.
\end{abstract}

\footnotetext{
* Autor/a correspondiente: Luciana Daniela Muñoz

Email: flga.lucianamunoz@gmail.com
}

Palabras clave:

Autopercepción de la voz; Voz; Mujeres trans; TWQV

\section{Keywords:} Self-perception of voice; Voice; Trans women; TWVQ 


\section{INTRODUCCIÓN}

La terminología en el ámbito de la salud para personas transgénero se encuentra evolucionando rápidamente, lo que implica la introducción constante de nuevos términos y cambios en las definiciones existentes. La Asociación Profesional Mundial para la Salud transgénero (WPATH por su sigla en inglés) es una organización internacional multidisciplinaria, promueve los cuidados basados en la evidencia, educación, investigación, políticas públicas y respeto a la salud transgénero. Incluso reconoce que pueden existir diferencias dependiendo de la cultura, comunidades y contextos (WPATH, 2012). Este artículo se enmarca en las definiciones planteadas por dicha asociación, que entrega una serie de conceptos que buscan facilitar una atención de calidad a las personas transgénero.

Cuando se habla de personas transgénero es necesario conocer conceptos tales como la Identidad de género, que se refiere a una percepción intrínseca de ser hombre, mujer o alguna alternativa de género. Se postula que esta percepción puede o no corresponder con el sexo asignado al nacer. Este último término se determina según la apariencia de los genitales externos, sexo cromosómico y hormonal. Una persona transgénero sería entonces aquella que su identidad de género difiere en diversos grados del sexo que se le asignó al nacer (WPATH, 2012). El Centro Educacional Nacional de la salud LGBTQIA+ (2020) complementa esta definición indicando que las personas transgéneros pueden tener identidad de género enmarcada dentro de una estructura binaria (femenina - masculina) o no binaria. Además, señala que se permite utilizar la abreviación "trans" para referirse a transgénero. De esta manera, se refiere a un hombre trans como una persona cuya identidad de género es masculina, una mujer trans como una persona cuya identidad de género es femenina y una persona no binaria como aquella que su identidad de género no se encuentra en la estructura dicotómica de femenino o masculino.

Cuando la diferencia entre identidad de género y sexo asignado al nacer genera molestia o incomodidad significativa en una persona se habla de Disforia de género (WPATH, 2012). Es importante mencionar que no todas las personas trans padecen de disforia de género (Adler et al., 2019).

Otro concepto importante para considerar es la expresión de género, la cual se refiere a la manera en que una persona comunica su identidad de género a través de la apariencia física, los gestos, los modos de hablar y los patrones de comportamiento con los demás. La comunicación, ya sea de forma verbal como no verbal, es un aspecto importante en la conducta humana y es parte de la expresión de género.

Una persona trans podría buscar apoyo en profesionales expertos para lograr una forma de comunicarse que le acomode con su identidad de género. En este contexto, la voz constituye un aspecto importante de la consulta (WPATH, 2012). Al respecto, la literatura sostiene que el principal grupo que asiste a consultar a entrenamiento vocal son las mujeres trans, razón por la cual existe mayor evidencia en torno a esta población (Davies et al., 2015).

Recomendaciones entregadas por la WPATH (2012) resaltan la importancia de que los especialistas en voz conozcan y respeten las preferencias comunicativas individuales de las mujeres que consultan por entrenamiento vocal. Recomiendan además no aconsejar adoptar comportamientos que las alejen de su comodidad y /o autenticidad. Sostienen que la experiencia de entrenamiento vocal mejora al comprender las preocupaciones y metas de las mujeres trans en torno a su voz. Es en este sentido, el especialista de la voz debe conocer la autopercepción de la voz de una mujer trans que quiere iniciar un entrenamiento vocal (WPATH, 2012).

La autopercepción de la voz es medida a través de un cuestionario de autorreporte. La información obtenida aporta datos importantes para el proceso de entrenamiento vocal propiamente tal, ya que la autopercepción de la voz puede no estar correlacionada a los posibles cambios acústico-perceptuales obtenidos. Se han reportado incongruencias entre las mediciones realizadas por el especialista en voz, las percepciones de la voz de oyentes evaluadores y la comunicación de las mujeres trans. Por lo que los resultados de la autopercepción de la voz ayudan al profesional a apreciar de mejor manera los ámbitos que son más problemáticos para la mujer trans en el momento de realizar el entrenamiento (Dacakis et al., 2016a, 2016b; Geneid et al., 2015).

Otros autores destacan que la evaluación de la autopercepción es importante para la pesquisa de dificultades de tipo fisiológicas tales como la pobre calidad y rango vocal restringidos. Dichas dificultades generan afectaciones emocionales, problemas de relaciones interpersonales tanto en el ámbito laboral como personal, evitación de actividades sociales, entre otras (Hancock, 2016; Hardy et al., 2014). La Organización Mundial de la Salud (OMS) reconoce que estos aspectos comunicativos, en especial aquellos relacionados con la función vocal y las consecuentes restricciones generadas en las actividades cotidianas, podrían facilitar o complicar la calidad de vida de una mujer trans, por lo 
que plantean la necesidad de ser consideradas tanto en la evaluación como en el entrenamiento vocal (Adler et al., 2019).

Con el fin de evaluar las posibles dificultades que presentan las mujeres trans debido a su voz nace el Transsexual Voice Questionnaire Male to Female (TVQmtf, por sus siglas en inglés). Este cuestionario evalúa la autopercepción de la voz de mujeres trans (Dacakis et al., 2013, 2016b; Davies \& Johnston, 2015).

Durante este mismo año, las autoras cambian el nombre del Transsexual Voice Questionnaire Male to Female a Trans Woman Voice Questionnaire (TWVQ, por sus siglas en inglés), por considerar que los términos "transexual" y "de hombre a mujer" ya no eran aceptables y que incluso podrían resultar ofensivos. Se realiza además la aclaración de que la modificación no compromete la validez del cuestionario (La Trobe University, 2020). Este artículo se utilizará el nombre adoptado en el año 2020, como una forma de apoyar dicho cambio (Trans Woman Voice Questionnaire o TWVQ).

A pesar de la evidente importancia de conocer la evaluación de autopercepción de la voz en mujeres trans, en nuestro país no existen estudios que indaguen sobre este aspecto. La consecuencia de la ausencia de información puede significar que solo se considere la evaluación y la opinión del especialista, sin tomar en cuenta lo que pueda declarar la mujer entrenada. Es por esta razón que el objetivo de este estudio fue describir la autopercepción de la voz de un grupo de mujeres, según el instrumento TWVQ.

\section{MÉTODO}

Este estudio es un diseño observacional, descriptivo correlacional de corte transversal con enfoque cuantitativo (Hernández Sampieri et al., 2014). Fue aprobado por el Comité de Ética (acreditado) de la Facultad de Medicina - Clínica Alemana Universidad del Desarrollo GP-70/2018.

\section{Participantes}

Las participantes fueron 30 mujeres trans de nacionalidad chilena, residentes en la ciudad de Santiago, Región Metropolitana, con un promedio de edad de 28 años (mínimo 18 años y máximo 57 años). El $60 \%$ de ellas había cursado educación media completa y el $40 \%$ restante eran profesionales o técnicas. Ninguna presentaba antecedentes de haber cursado cirugía vocal para modificar el tono de su voz y/o haber presentado patologías laríngeas, auditivas $\mathrm{y} / \mathrm{o}$ respiratorias crónicas diagnosticadas por otorrinolaringólogo [ORL].
Se seleccionaron mediante un muestreo no probabilístico de bola de nieve. Este consistió en un primer contacto en donde se invitó a participar a tres mujeres trans a través de las redes sociales: Facebook e Instagram. Ellas después de aceptar integrar la muestra del estudio, ayudaron a contactar a otras mujeres que cumplieran con los criterios de inclusión y exclusión. Así, se invitó a 34 personas, de las cuales 30 estaban dispuestas a colaborar. Todas firmaron un consentimiento informado en donde se aseguró la confidencialidad y anonimato de la información.

\section{Instrumentos}

Los instrumentos que se aplicaron fueron dos. El primero es el Cuestionario de antecedentes clínicos, el cual entrega información relacionada con: transición de género, asistencia y tiempo de terapia hormonal, cirugía de afirmación de género y asistencia al especialista de la voz. El segundo, es el cuestionario Trans Woman Voice Questionnaire [TWVQ] que está validado por Dacakis et al. (2016a). Está traducido al español por Mora et al. (2018) y su nombre en este idioma es Cuestionario de voz transexual de hombre a mujer.

El cuestionario TWVQ se encuentra estructurado en dos partes. La primera, consta de 30 preguntas que abordan las consecuencias psicosociales que vivencian diariamente las mujeres trans en relación a su voz, a través de una escala Likert de 4 puntos con las siguientes opciones de respuesta: $1=$ nunca $o$ muy raramente, $2=\mathrm{a}$ veces, $3=$ a menudo y $4=$ frecuentemente o siempre; asignando un puntaje total con un mínimo de 30 y un máximo de 120 puntos. De acuerdo a lo planteado por las autoras del instrumento, la valoración considera que cuanto más alta sea la puntuación, mayores serán las dificultades percibidas y los impactos psicosociales que tiene la voz de la evaluada (Dacakis et al., 2016b).

Dacakis et al. (2016b) propuso para un análisis detallado, dos grupos o ítems: "funcionamiento vocal" (14 preguntas) y el de "participación social" (12 preguntas), quedando 4 preguntas sin clasificar. Para una mejor interpretación y reporte de los resultados, los autores Bultynck et al. (2017) y Bultynck et al., (2020) aplicaron un análisis factorial a las primeras 30 preguntas, lo que permitió distribuir las interrogantes en 3 grupos o ítems (Tabla 1). El ítem de ansiedad y evitación está compuesto por preguntas sobre sentirse ansiosa o incómoda en contextos laborales o sociales (11 preguntas; puntuación mínima $=11$, puntuación máxima $=44)$. El ítem de identidad vocal abarca preguntas sobre disconformidad producto de las características vocales y la incongruencia entre la identidad de género y su expresión (8 preguntas; puntuación mínima $=8$, puntuación 
máxima =32). Por último, el ítem función vocal incluye preguntas sobre las características de la voz (11 preguntas; puntuación mínima $=11$, puntuación máxima $=44)$.

Tabla 1. Distribución de preguntas según ítem del TWVQ.

\section{Ítem de ansiedad y evitación}

Siento ansiedad cuando sé que tengo que utilizar mi voz.

Evito usar el teléfono debido a mi voz.

Estoy tensa al hablar con otros debido a mi voz.

Me siento incómoda cuando hablo con amigos, vecinos y familiares debido a mi voz.

Evito hablar en público debido a mi voz.

Me siento frustrada cuando intento cambiar mi voz.

Las dificultades con mi voz restringen mi vida social.

Mi voz restringe el tipo de trabajo que hago.

Soy menos extrovertida debido a mi voz.

Me siento cohibida por cómo los extraños perciben mi voz.

Me siento discriminada a causa de mi voz.

Ítem de identidad vocal

Mi voz me hace sentir menos femenina de lo que me gustaría.

El tono de mi voz es demasiado grave.

Mi voz es un obstáculo para vivir como mujer.

Mi voz dificulta que me identifiquen como una mujer.

Cuando me río sueno como un hombre.

Mi voz no se corresponde con mi aspecto físico.

Siento que mi voz no refleja mi 'verdadero yo'.

Me angustia que me perciban como un hombre debido a mi voz.

Ítem de función vocal

La gente tiene dificultades para oírme en un sitio ruidoso.

El tono de mi voz es impredecible.

Mi voz suena áspera, ronca o seca cuando intento hablar con una voz femenina.

El tono de mi voz no varía lo suficiente cuando hablo.

Mi voz suena artificial.

Tengo que concentrarme para lograr que mi voz suene como quiero que lo haga.

Cuando no prestó atención, el tono de mi voz se vuelve más grave.

Hago mucho esfuerzo para producir mi voz.

Mi voz se cansa rápidamente.

Mi voz falla a mitad de la conversación.

El rango de tono de mi voz es limitado.
La segunda parte del cuestionario TWVQ consta de 2 preguntas, correspondientes a lo que las autoras denominan valoración global de la voz actual y de la voz ideal. Las opciones que tienen de respuesta son: muy femenina, algo femenina, neutra, algo masculina y muy masculina (Dacakis et al., 2016b).

\section{Procedimientos de evaluación}

El Cuestionario de antecedentes clínicos se les administró verbalmente a las participantes, mientras que el TWVQ fue entregado físicamente para su autoaplicación.

\section{Análisis estadístico}

Los datos se analizaron utilizando el software IBM SPSS Statistics, versión 22. Inicialmente se realizó un análisis estadístico descriptivo. Para las variables cuantitativas se calcularon promedios, mediana, valores mínimos, máximos y desviación estándar de la primera parte del TWVQ que alude a los puntajes obtenidos para las 30 preguntas del instrumento y para cada uno de los ítems. Para la segunda parte del TWVQ sobre percepción de voz actual y la voz ideal, se calcularon frecuencias y porcentajes de los resultados obtenidos.

En el caso del cuestionario de antecedentes clínicos en el tiempo de terapia hormonal, se calcularon promedios, valores mínimos, máximos y desviación estándar. Para las variables: terapia hormonal, cirugía de afirmación de género, asistencia al especialista de la voz, se calcularon frecuencias y porcentajes.

Luego, se analizaron los puntajes obtenidos del TWVQ total y por ítems, según características de la muestra de estudio que alude a la edad, al tiempo de terapia hormonal y a la asistencia al especialista de la voz. En efecto, en primer lugar se aplicó la prueba de Shapiro-Wilk a los puntajes obtenidos del instrumento principal, reportando una distribución normal. Por consiguiente, para los puntajes de las variables cuantitativas de edad y tiempo de terapia hormonal se empleó la prueba de Correlación de Pearson. Para las variables cualitativas, como la asistencia al especialista de la voz, se utilizó la prueba $t$ de Student, considerando un valor $\mathrm{p}<0,05$.

No se realizaron análisis con las variables terapia hormonal y cirugía de afirmación de género, ya que las respuestas fueron $100 \%$ unánimes. Así, todas estaban en tratamiento hormonal y ninguna se había sometido a cirugía. 


\section{RESULTADOS}

\section{Participantes}

El análisis descriptivo del cuestionario de antecedentes clínicos mostró que el tiempo de terapia hormonal de las usuarias era de 29.33 meses en promedio, con un mínimo de 1 mes y máximo 96 meses. También, informó que un 43,3\% asistió al especialista de la voz, con el fin de entrenar sus características vocales para una mayor conformidad entre su identidad y su expresión de género. Algunas participantes incluso aludieron a la "feminización vocal".

En la Tabla 2 se exponen los estadísticos descriptivos de la primera parte del instrumento que mide la autopercepción de la voz. De forma más específica el ítem de función vocal es el que muestra una mayor dificultad, posteriormente le sigue el ítem de ansiedad y evitación y finalmente el de identidad vocal.

Tabla 2. Estadísticos descriptivos de los puntajes obtenidos del TWVQ.

\begin{tabular}{|c|c|c|c|c|c|c|}
\hline & Promedio & Mediana & Mínimo & Máximo & Desviación estándar & $\mathrm{N}^{\circ}$ \\
\hline Puntaje total TWVQ & 61,47 & 63 & 36 & 100 & 16,972 & 30 \\
\hline ítem ansiedad y evitación & 21,07 & 21 & 11 & 35 & 6,992 & 30 \\
\hline ítem función vocal & 20,6 & 21 & 12 & 36 & 6,173 & 30 \\
\hline Ítem identidad vocal & 20,9 & 22 & 10 & 30 & 6,105 & 30 \\
\hline
\end{tabular}

Total TWVQ (30 preguntas; puntuación mínima $=30$, puntuación máxima $=120)$, ítem de ansiedad y evitación $(11$ preguntas; puntuación mínima $=11$, puntuación máxima $=44)$, ítem de identidad vocal $(8$ preguntas; puntuación mínima $=8$, puntuación máxima $=32)$ e ítem función vocal $(11$ preguntas; puntuación mínima $=11$, puntuación máxima $=44)$.

En la Tabla 3 se muestran los estadísticos descriptivos de la segunda parte del TWVQ, referidos a la voz actual y a la voz ideal. Si se observan los porcentajes más altos en cada una de las valoraciones, se establece una consistencia entre la propia voz autopercibida como "muy masculina" y la característica vocal ideal "muy femenina" reportada por las participantes.

Tabla 3. Estadísticos descriptivos de la valoración global de la voz actual y voz ideal.

\begin{tabular}{|c|c|c|c|c|c|c|}
\hline & Muy femenina & Algo femenina & Neutra & Algo masculina & Muy masculina & Total \\
\hline \multicolumn{7}{|c|}{ Valoración global de "mi voz actual" } \\
\hline Frecuencia & 1 & 6 & 3 & 12 & 8 & 30 \\
\hline Porcentaje & 3,3 & 20 & 10 & 40 & 26,7 & 100 \\
\hline \multicolumn{7}{|c|}{ Valoración global de "mi voz ideal" } \\
\hline Frecuencia & 8 & 15 & 6 & 1 & 0 & 30 \\
\hline Porcentaje & 26,7 & 50 & 20 & 3,3 & 0 & 100 \\
\hline
\end{tabular}

En la Tabla 4 se expone la correlación de los puntajes obtenidos del TWVQ, según el tiempo de terapia hormonal. El ítem de ansiedad y evitación es el único que reportó una posible relación con un $\mathrm{p}=0,017$.
Tabla 4. Puntajes obtenidos del TWVQ según el tiempo de terapia hormonal.

\begin{tabular}{lc}
\hline Puntajes obtenidos del TWVQ & Pearson Correlación \\
\cline { 2 - 2 } Puntaje total TWVQ & 0,117 \\
ítem ansiedad y evitación & $0,017 *$ \\
ítem función vocal & 0,094 \\
Ítem identidad vocal & 0,109 \\
\hline
\end{tabular}

* Resultados significativos $\mathrm{p}<0,05$. 
En la Tabla 5 se observan las diferencias de los promedios obtenidos en los puntajes del TWVQ, entre las que asistieron al especialista de la voz y las que no lo efectuaron. El ítem de función vocal es el que presenta una diferencia con un $\mathrm{p}=0,009$.

Tabla 5. Puntajes obtenidos del TWVQ según asistencia al especialista de la voz.

\begin{tabular}{|c|c|c|c|c|c|}
\hline Puntajes obtenidos del TWVQ & Asistencia al especialista de la voz & $\mathrm{N}^{\circ}$ & Promedio & Deviación estándar & Prueba t de Student \\
\hline \multirow[t]{2}{*}{ Puntaje total TWVQ } & Sí & 13 & 64,92 & 20,127 & \multirow{2}{*}{0,363} \\
\hline & No & 17 & 58,82 & 14,183 & \\
\hline \multirow[t]{2}{*}{ ítem ansiedad y evitación } & Sí & 13 & 21,31 & 8,45 & \multirow{2}{*}{0,878} \\
\hline & No & 17 & 20,88 & 5,915 & \\
\hline \multirow[t]{2}{*}{ ítem función vocal } & Sí & 13 & 24 & 6,298 & \multirow{2}{*}{$0,009 *$} \\
\hline & No & 17 & 18 & 4,77 & \\
\hline \multirow[t]{2}{*}{ Ítem identidad vocal } & Sí & 13 & 19,08 & 7,205 & \multirow{2}{*}{0,181} \\
\hline & No & 17 & 22,29 & 4,883 & \\
\hline
\end{tabular}

* Resultados significativos $\mathrm{p}<0,05$.

\section{DISCUSIÓN}

El objetivo del estudio fue describir la autopercepción de la voz de un grupo de mujeres trans de la Región Metropolitana, según el instrumento TWVQ. Se empleó un cuestionario dado que la WPATH (2012) recomienda este tipo de herramientas, ya que ayudan a comprender al terapeuta la influencia de la voz sobre los diversos ámbitos de la vida cotidiana.

Según los resultados obtenidos en este estudio, para el puntaje total del TWVQ (promedio 61,47 puntos y mediana 63 puntos) existe un impacto psicosocial en las participantes, según su autopercepción de la voz. De acuerdo con Diamant \& Amir (2020), la autoevaluación vocal se ve afectada por elementos que comprenden la autosatisfacción y experiencia personal.

Para los terapeutas la existencia de un impacto psicosocial, de acuerdo con la autopercepción vocal de la usuaria, es determinante en términos generales, pero inespecífica para el abordaje. Es por ello que los resultados se analizaron en base a los 3 ítems planteados por Bultynck et al. (2017) y Bultynck et al. (2020). Según la muestra del estudio, la dificultad observada para el total del TWVQ está dada primeramente por el ítem de función vocal, ya que es el que muestra una mayor dificultad. Posteriormente, le sigue el ítem de ansiedad y evitación, finalizando con el de identidad vocal.

En los resultados del ítem de ansiedad y evitación, las mujeres trans experimentan incomodidad o ansiedad debido a su voz. Estas sensaciones las vivencian en el contexto laboral y en el social, teniendo como consecuencia la restricción de su participación en dichos contextos. Lo anterior concuerda con lo que plantean Hardy et al. (2014) y Hancock (2016) conforme a la CIF. Por su parte, Gómez-Raya (2018) agrega que también afecta el contexto familiar (Davies et al., 2006).

Davies \& Johnston (2015) exponen que el puntaje de las preguntas que agrupa el ítem de ansiedad y evitación está dado por el nivel de actividad social de la mujer trans. Una mujer puede presentar un puntaje bajo en dos casos muy distintos. El primero al tener una vida social activa y saludable, no experimentando dificultades en contexto social a costa de su voz y el segundo por estar socialmente aislada, teniendo rara vez interacciones sociales a causa del temor al ridículo o a la violencia, no percibiendo su voz como un problema.

En cuando a los resultados obtenidos para el ítem de identidad vocal, las participantes muestran una alta inconformidad entre la identidad de género y la expresión de género, por el uso de su voz. En la literatura existe una descripción bastante robusta (basada en opiniones de mujeres trans, apreciación de expertos y evidencia empírica) que justifica que ellas concurran a especialistas de la voz para un posible entrenamiento vocal (Adler et al., 2012; Bultynck et al., 2017; Cárdenas et al., 2019; Dacakis et al., 2017; Davies et al., 2015; Diamant \& Amir, 2020; Gómez-Raya, 2018; Hyung-Tae, 2020; Leyns et al., 2021; Shefcik \& Tsai, 2021).

Respecto al ítem de función vocal, el funcionamiento de la voz y sus características son una dificultad percibida en las mujeres trans del estudio, lo que significaría una limitación en su actividad comunicativa. Hardy et al. (2014) aclaran, a partir de la CIF, que 
las mujeres trans presentan en su vida diaria continuas limitaciones en su comunicación, ya sea a nivel verbal o no verbal. Además, describen elementos relacionados a la concentración, esfuerzo y cansancio vocal que presentan las usuarias para mantener el tono de voz deseado.

Es necesario destacar que en los estudios anteriores no se analizaron en profundidad la sección correspondiente a la percepción de la voz actual y de la voz ideal. No obstante, en la muestra del presente estudio se observa en los resultados valores predominantes, para la voz actual como "algo masculina" y para la voz ideal como "algo femenina". También, existe una igualdad en porcentajes para la voz actual como "muy masculina" y la voz ideal como "muy femenina", en un $26,7 \%$. Esta información entrega al terapeuta las aspiraciones de la mujer trans en relación a su voz, para que su expresión de género sea congruente con la identidad de género.

Lo anterior se encuentra en línea con lo que plantea la WPATH (2012) en base a la información que debe entregar un cuestionario de autopercepción y expone que los especialistas en voz deben respetar y conocer las preferencias comunicativas individuales de las personas que consultan por sus servicios. Se sostiene que la experiencia con el entrenamiento vocal mejora al comprender las preocupaciones y metas de las personas en torno a su voz (Davies, 2017; Davies et al., 2015; Davies \& Johnston, 2015; Leyns et al., 2021).

El puntaje del ítem de ansiedad y evitación del uso de la voz se relacionó con el tiempo de terapia hormonal cursado. Así, mientras más tiempo ha transcurrido en la terapia disminuye la ansiedad y la necesidad de evitar usar la voz. Esta relación concuerda con lo hallado por los autores Bultynck et al. (2017) que advirtieron una mejora del ítem a partir de los meses de terapia hormonal cursada. Sin embargo, plantean que no existe una relación lineal respecto a la disminución de los niveles de testosterona y la autopercepción de la voz.

El puntaje del ítem de función vocal varía según la asistencia al especialista de la voz, para un entrenamiento vocal. Esto coincide con lo encontrado en la literatura que indica que asistir a entrenamiento vocal, con la consecuente mejoría de la función vocal, impacta positivamente en su calidad de vida. Ello debido a que las expresiones verbales de las mujeres trans alcanzan mayor naturalidad (Davies et al., 2006; Diamant \& Amir, 2020; Gelfer \& Tice, 2013; Gelfer \& Van Dong, 2013; Hancock \& Pool, 2017; Jones et al., 2019; Leyns et al., 2021; Owen \& Hancock, 2011).

Respecto a la naturalidad de la comunicación de las mujeres trans, Mills et al. (2017) proponen una serie de aspectos que contribuyen a alcanzar esta naturalidad. Dichos aspectos son: elementos relacionados a la flexibilidad tonal, aumento de la frecuencia fundamental, correcto empleo de las cavidades de resonancia del tracto, apoyado de los elementos no verbales.

Finalmente, en este estudio se destacan tres limitaciones: la primera limitación alude al reducido tamaño de la muestra. A pesar del pequeño número de participantes, se obtuvieron resultados relevantes, que podrían ser reforzados en investigaciones que incluyan muestras con mayor tamaño. La segunda limitación refiere al procedimiento utilizado para detectar posibles patologías del tipo laríngeas, auditivas y/o respiratorias crónicas, que afecten la voz. Esto debido a que la mayoría de las participantes relataron limitaciones socioeconómicas para acceder a profesional especializado. La última limitación se relaciona con el análisis del instrumento TWVQ. Dacakis et al. (2016a, 2016b) proponen rangos de mínimos y máximos globales, no clasificando jerárquicamente a la persona, en función a la autopercepción de su voz. Además, para el análisis detallado del instrumento, no incluyen las 30 preguntas en la distribución de ítems.

\section{CONCLUSIÓN}

Los resultados de este estudio sugieren que la autopercepción de la voz se ve afectada en primer lugar por el ítem de función vocal, posteriormente por el ítem de ansiedad y evitación y por último por el de identidad vocal. Los resultados también proporcionan evidencia preliminar de que la autopercepción de la voz podría asociarse con la asistencia al especialista de la voz para un entrenamiento vocal y con el tiempo que ha transcurrido de terapia hormonal. Por último, el estudio proporciona una forma empírica de analizar detalladamente el TVWQ, en base a la propuesta de tres ítems.

\section{REFERENCIAS}

Adler, R., Hirsch, S., \& Mordaunt, M. (2012). Voice and Communication Therapy for the Transgender/Transsexual Client: A Comprehensive Clinical Guide. Plural Publishing.

Adler, R., Hirsch, S., \& Pickering, J. (2019). Voice and Communication Therapy for the Transgender/Gender Diverse Client: A Comprehensive Clinical Guide (3era ed.). Plural Publishing.

Bultynck, C., Pas, C., Defreyne, J., Cosyns, M., Heijer, M. den, \& T'Sjoen, G. (2017). Self-perception of voice in transgender persons during cross-sex hormone therapy. The Laryngoscope, 127(12), 2796-2804. https://doi.org/10.1002/lary.26716 
Bultynck, C., Pas, C., Defreyne, J., Cosyns, M., \& T’Sjoen, G. (2020). Organizing the voice questionnaire for transgender persons. International Journal of Transgender $\quad$ Health, 21(1), 89-97. https://doi.org/10.1080/15532739.2019.1605555

Cárdenas, Y., Campo, C., Fernández, V., Escobedo, J., Inchuchala, J., Delgado, J. P., Ramírez, E. J., \& Gómez, C. (2019). Intervención fonoaudiológica para la feminización de la voz en una persona transgénero (MTF): Estudio de caso. Revista Chilena de Fonoaudiología, 18, 1-15. https://doi.org/10.5354/07194692.2019.55328

Dacakis, G., Davies, S., Oates, J., Douglas, J., \& Johnston, J. (2013). Development and Preliminary Evaluation of the Transsexual Voice Questionnaire for Male-toFemale Transsexuals. Journal of Voice, 27(3), 312-320. https://doi.org/10.1016/j.jvoice.2012.11.005

Dacakis, G., Oates, J., \& Douglas, J. (2016a). Exploring the validity of the Transsexual Voice Questionnaire (male-to-female): Do TVQMtF scores differentiate between $\mathrm{MtF}$ women who have had gender reassignment surgery and those who have not? International Journal of Transgenderism, 17(3-4), 124-130. https://doi.org/10.1080/15532739.2016.1222922

Dacakis, G., Oates, J., \& Douglas, J. (2016b). Further Evidence of the Construct Validity of the Transsexual Voice Questionnaire (TVQMtF) Using Principal Components Analysis. Journal of Voice, 31(2), 142-148. https://doi.org/10.1016/j.jvoice.2016.07.001

Dacakis, G., Oates, J., \& Douglas, J. (2017). Associations between the Transsexual Voice Questionnaire (TVQMtF) and self-report of voice femininity and acoustic voice measures. International Journal of Language \& Communication Disorders, 52(6), 831-838. https://doi.org/10.1111/1460-6984.12319

Davies, S. (2017). The Evidence Behind the Practice: A Review of WPATH Suggested Guidelines in Transgender Voice and Communication. Perspectives of the ASHA Special Interest Groups, 2(10), 64-73. https://doi.org/10.1044/persp2.SIG10.64

Davies, S. M., \& Johnston, J. R. (2015). Exploring the validity of the Transsexual Voice Questionnaire for male-to-female transsexuals. Canadian Journal of Speech-Language Pathology and Audiology, 39(1), 40-51.

Davies, S., Papp, V. G., \& Antoni, C. (2015). Voice and Communication Change for Gender Nonconforming Individuals: Giving Voice to the Person Inside. International Journal of Transgenderism, 16(3), 117-159. https://doi.org/10.1080/15532739.2015.1075931

Davies, S., RSLP-C, \& Goldberg, J. (2006). Clinical Aspects of Transgender Speech Feminization and Masculinization. International Journal of Transgenderism, 9(3-4), 167-196. https://doi.org/10.1300/J485v09n03_08

Diamant, N., \& Amir, O. (2020). Examining the voice of Israeli transgender women: Acoustic measures, voice femininity and voice-related quality-of-life. International Journal of Transgender Health, 22(3), 281-293. https://doi.org/10.1080/26895269.2020.1798838

Gelfer, M. P., \& Tice, R. M. (2013). Perceptual and acoustic outcomes of voice therapy for male-to-female transgender individuals immediately after therapy and 15 months later. Journal of Voice: Official Journal of the Voice Foundation, 27(3), 335-347. https://doi.org/10.1016/j.jvoice.2012.07.009
Gelfer, M. P., \& Van Dong, B. R. (2013). A preliminary study on the use of vocal function exercises to improve voice in male-to-female transgender clients. Journal of Voice: Official Journal of the Voice Foundation, 27(3), 321-334. https://doi.org/10.1016/j.jvoice.2012.07.008

Geneid, A., Rihkanen, H., \& Kinnari, T. J. (2015). Long-term outcome of endoscopic shortening and stiffening of the vocal folds to raise the pitch. European Archives of Oto-Rhino-Laryngology, 272(12), 3751-3756. https://doi.org/10.1007/s00405-015-3721-7

Gómez-Raya, A. (2018). Intervención logopédica en la feminización de la voz en transexuales: Revisión bibliográfica. Revista de Investigación en Logopedia, 8(1), 21-42. https://doi.org/10.5209/RLOG.59528

Hancock, A. B. (2016). An ICF Perspective on Voice-related Quality of Life of American Transgender Women. Journal of Voice: Official Journal of the Voice Foundation, 31(1), 115.e1-115.e8. https://doi.org/10.1016/j.jvoice.2016.03.013

Hancock, A. B., \& Pool, S. F. (2017). Influence of Listener Characteristics on Perceptions of Sex and Gender. Journal of Language and Social Psychology, 36(5), 599-610. https://doi.org/10.1177/0261927X17704460

Hardy, T. L. D., Boliek, C. A., Wells, K., \& Rieger, J. M. (2014). The ICF and Male-to-Female Transsexual Communication. International Journal of Transgenderism, 14(4), 196-208. https://doi.org/10.1080/15532739.2014.890561

Hernández Sampieri, R., Fernández Collado, C., \& Baptista Lucio, M. P. (2014). Metodología de la investigación (6ta ed.). McGraw-Hill.

Hyung-Tae, K. (2020). Vocal Feminization for Transgender Women: Current Strategies and Patient Perspectives. International Journal of General Medicine, 13, 43-52. https://doi.org/10.2147/IJGM.S205102

Jones, B., Bouman, W. P., Haycraft, E., \& Arcelus, J. (2019). The Gender Congruence and Life Satisfaction Scale (GCLS): Development and validation of a scale to measure outcomes from transgender health services. International Journal of Transgenderism, 20(1), 63-80. https://doi.org/10.1080/15532739.2018.1453425

La Trobe University. (2020). Voice. The Trans Woman Voice Questionnaire $(T W V Q)$. Resources. https://www.latrobe.edu.au/communication-clinic/resources

Leyns, C., Papeleu, T., Tomassen, P., T’Sjoen, G., \& D'haeseleer, E. (2021). Effects of speech therapy for transgender women: A systematic review. International Journal of Transgender Health, 1-21. https://doi.org/10.1080/26895269.2021.1915224

Mills, M., Stoneham, G., \& Georgiadou, I. (2017). Expanding the evidence: Developments and innovations in clinical practice, training and competency within voice and communication therapy for trans and gender diverse people. International Journal of Transgenderism, 18(3), 328-342. https://doi.org/10.1080/15532739.2017.1329049

Mora, E., Carrillo, A., Giribet, A., Becerra, A., Lucio, M. J., \& Cobeta, I. (2017). Translation, Cultural Adaptation, and Preliminary Evaluation of the Spanish Version of the Transgender Voice Questionnaire for Male-to-Female Transsexuals (TVQ MtF). Journal of Voice, 32(4), 514.e1-514.e6. https://doi.org/10.1016/j.jvoice.2017.05.012

National LGBT Health Education Center. (2020). LGBTQIA+ Glossary of Terms for Health Care Teams. https://www.lgbtqiahealtheducation.org/wpcontent/uploads/2020/10/Glossary-2020.08.30.pdf 
Owen, K., \& Hancock, A. (2011). The Role of Self- and Listener Perceptions of Femininity in Voice Therapy. International Journal of Transgenderism, 12(4), 272-284. https://doi.org/10.1080/15532739.2010.550767

Shefcik, G., \& Tsai, P.-T. (2021). Voice-related Experiences of Nonbinary Individuals (VENI) Development and Content Validity. Journal of Voice. https://doi.org/10.1016/j.jvoice.2020.12.037
World Professional Association for Transgender Health [WPATH]. (2012). Standards of Care for the Health of Transsexual, Transgender, and GenderConforming People. https://www.wpath.org/publications/soc 